\title{
Stochastic Topology Optimization Based on Level-Set Method
}

\author{
Yuki Hidaka, Takahiro Sato, Hajime Igarashi \\ Graduate school of Information Science and Technology \\ Hokkaido University \\ Sapporo, Japan \\ hidaka@em-si.eng.hokudai.ac.jp
}

\author{
Kota Watanabe \\ Graduate school of Engineering \\ Muroran Institute of Technology \\ Muroran, Japan \\ k-wata@mmm.muroran-it.ac.jp
}

\begin{abstract}
The level-set method has been shown suitable for topology optimization because it can find optimal shapes with smooth boundaries and non-porous material regions. In the level-set method, however, optimization is conducted based on the gradient method. For this reason, the solutions obtained by the level-set method tend to fall into local optima. In this work, the simulated annealing is introduced into the level-set method to solve this problem. In order to test the present method, the shape of the flux barrier in the rotor of an interior permanent magnetic motor (IPM-motor) is optimized. It is shown that the resultant properties are better than those obtained by the deterministic level-set method.
\end{abstract}

Keywords-level-set method; simulated annealing; topology optimization; IPM-motor;

\section{INTRODUCTION}

Shape optimization has widely been used for the development of electromagnetic devices. There are two different approaches for the shape optimizations, namely, parameter and topology optimizations. In the parameter optimization, device shapes are represented with parameters and are optimized by changing them. On the other hand, device shapes are treated as binary pixel images and are optimized by changing the pixel values in the topology optimization based on the on-off method. This method can lead to novel shapes which cannot be found by engineers. However, the device shapes obtained by the on-off method are often have jagged boundaries and many porous material regions because of the huge search spaces. For this reason, it is difficult to obtain smooth shapes adequate for productions when using the conventional on-off method [1][2].

The level-set based topology optimization can solve these problems. The level-set method is an optimization method to search for optimal material boundaries through implicit function called the level-set function. This method can easily find solutions with smooth boundary and nonporous material region. However, the level-set functions are updated to minimize the objective function on the basis of the gradient method. For this reason, the resultant shapes strongly depend on the initial shape. This means that the level-set method tends to converge to one of the local optima if the initial boundaries are not appropriate [3][4].

In this work, the simulated annealing (SA) is introduced to the level-set based topology optimization to solve above problems. Because of the stochastic change in the gradient descent, the present method has higher search ability than conventional method. In this work, the present method is applied to the rotor design of an IPM-motor. It is shown that the present method can find solutions with better performance than those obtained by the conventional method [5].

\section{OPTIMIZATION METHOD}

\section{A. Level-Set Method}

In the level-set method material shapes are represented using implicit function called the level-set function. The level-set function $\phi(x)$ takes the following values

$$
\phi(x)\left\{\begin{array}{cc}
>0 & x \in \Omega \\
=0 & x \in \partial \Omega \mathrm{I} D \\
<0 & x \in D \backslash \Omega
\end{array}\right.
$$

where $\boldsymbol{x}$ is the position vector, and $D, \Omega, D \backslash \Omega$, represents design, material and air regions, respectively. In the optimization, $\phi(x)$ is changed to minimize the objective function $F(\phi)$ where its temporal change is governed by

$$
\frac{\partial \phi(\boldsymbol{x}, t)}{\partial t}+V_{N}(\boldsymbol{x}, t)=0
$$

where $V_{N}(\boldsymbol{x}, t)$ denotes the normal velocity of boundary which is defined by

$$
V_{N}(x, t)=\frac{\mathrm{d} F}{\mathrm{~d} \phi}
$$

In the conventional method, $\mathrm{d} F / \mathrm{d} \phi$ is determined using the adjoint variable method which is one of the gradient methods [3][4]. For this reason, the conventional level-set method tends to converge to local optima.

\section{B. Level-Set Method Coupled with Simulated Annealing}

As mentioned above, the level-set function is updated by the gradient-based method in the conventional approach. For this reason, the solution may fall into one of the local optima. In order to solve this problem, it would be effective to introduce stochastic optimization techniques. The evolutionary approaches such as genetic and immune algorithms have widely been used in the stochastic optimizations. Although they have great ability to find the 
global optima, it seems to be difficult to combine them with level-set method because they are population-based algorithms. On the other hand, SA searches for the global optima on the basis of local search with heating and controlled cooling processes. Thus, this method can be combined easily with the level-set method.

The conventional SA involves cooling process and local search operation. It is known, however, these process take long time for convergence [5]. For this reason, we employ here the simplified SA in which the update descent of the level-set functions $V_{N}$, is randomly reversed as follows:

$$
V_{N}^{\prime}(\boldsymbol{x}, t)=\left\{\begin{array}{cc}
V_{N}(\boldsymbol{x}, t) & (r>P) \\
\alpha V_{N}(\boldsymbol{x}, t) & (r<P)
\end{array}\right.
$$

where $\alpha$ takes values in the domain $[-1,1]$. Moreover, $r$ is random number with interval $[0,1]$ and $p$ is the probability in directional reversal obtained by

$$
P=\exp \left(\frac{f^{\prime}-f}{T_{n}}\right)
$$

where $f$ and $f$ 'are the values of the object functions before and after the update, respectively, and $T_{n}$ is the temperature parameter obtained by

$$
T_{n}=R T_{n-1}
$$

where $R$ is the cooling rate of $T_{n}$.

Thanks to this stochastic change, it is expected that the present method finds solutions with better performance in comparison with the conventional level-set method.

\section{NUMERICAL RESULT}

\section{A. Optimization Problem}

In this work, to test the present method, we optimize the shape of the flux barrier in the rotor of an IPM-motor shown in Fig. 1(a) [6]. Due to the symmetry in this model, $1 / 4$ of the whole model is analyzed. The object function $F$ is defined to maximize the torque average and minimize the torque ripple as follows:

$$
F(\phi)=-\frac{T_{\text {average }}}{T_{\text {average }}^{0}}+W \frac{T_{\text {ripple }}}{T_{\text {ripple }}^{0}}
$$

where $W$ is a weighting coefficient, $T_{\text {average }}$ and $T_{\text {ripple }}$ are the torque average and ripple. Moreover, $T_{\text {average }}$ and $T_{\text {average }}^{0}$ represents reference values of torque and torque ripple, $3.971 \mathrm{Nm}$ and 0.353 respectively, of the reference model shown in Fig. 1(b). In this work, $T_{\text {ripple }}$ is defined as follows:

$$
T_{\text {ripple }}=\frac{T_{\max }-T_{\min }}{T_{\text {average }}}
$$

where $T_{\max }$ and $T_{\min }$ are the maximum and minimum values of the torque.

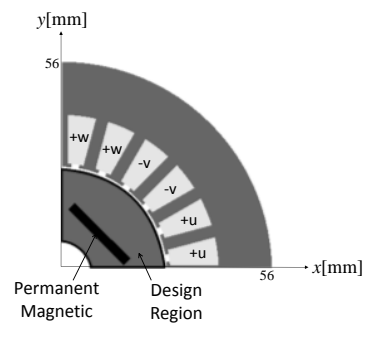

(a). Motor model

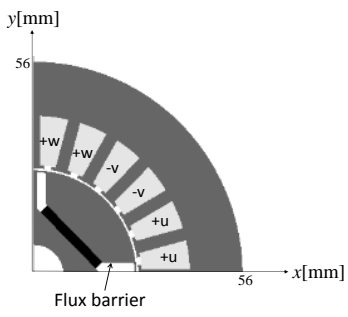

(b). Reference model
Fig. 1 Optimization model

\section{B. Numerical Result}

The solutions obtained by the conventional level-set method and the present method are shown in Fig. 2. The values of torque average, torque ripple and object function are summarized in Table. I. From these results, we can see the present method can find solution more better performance than the conventional level-set method.

In the full paper, optimization process will be reported in detail and other devices are optimized by the present method.

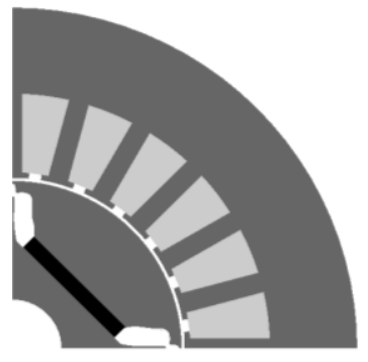

(a). Conventional method

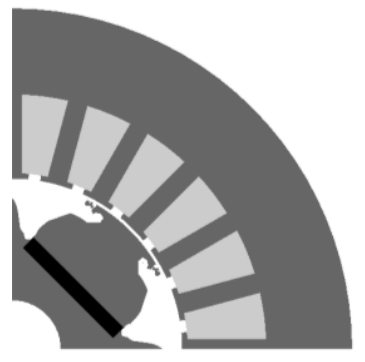

(b). Present method
Fig. 2 Optimized shapes

TABLE I.OPTIMIZATION RESULTS

\begin{tabular}{lcc}
\hline \hline & Conventional method & Present method \\
\hline$T_{\text {average }}(\mathrm{Nm})$ & 3.907 & 4.499 \\
$T_{\text {ripple }}$ & 0.227 & 0.131 \\
Object function & -0.426 & -0.794 \\
\hline
\end{tabular}

\section{REFERENCES}

1] K. Watanabe, F. Campelo, Y.Iijima, K. Kawano, T. Matsuo, T. Mifune, H.Igarashi, "Optimization of Inductors Using Evolutionary Algorithms and Its Experimental Validation", IEEE Transactions on Magnetics, Volume: 46 Issue:8, pp. 3393-3396, 2010

[2] J. Cros, P. Viarouge, M. T. Kakhki, "Design and Optimization of Soft Magnetic Composite Machines With Finite Element Methods", IEEE, Trans, Magn,, Vol, 47, No, 10, pp, 4384-4390, 2011.

[3] Stanley Osher, Ronald Fedkiw, Level Set Methods and Dynamic Implicit Surfaces, Springer Science+Business Media, LLC, 2003.

[4] Sang-in Park, Seungjae Min, Shintaro Yamasaki, Shinji nishiwaki, and Jeondhoon Yoo, "Magnetic Actuator Design Using Level Set Based Topology Optimization”, IEEE Trans, Magn,, vol,44, no,11, pp, 4037-4040, 2008.

[5] Wang Youhua, Yan Weili and Zhang Guansheng, "Adaptive Simulated Annealing for the Optimal Design of Electromagnetic Devices", IEEE Trans. Magn, vol.32, no.3, 1996, pp.1214-1217.

[6] Technical report of the institute of electrical engineering of Japan, industry application society, No. 776, 2000. 